\title{
THE APPLICABILITY OF FUNCTIONAL ANALYSIS IN FIXED PROSTHETICS
}

\author{
CRISTINA ADRIANA DAHM TĂTARU ${ }^{1}$
}

I "Lucian Blaga” University of Sibiu

Keywords: temporomandibular, overdenture, dentistry, prosthetic, implant

\begin{abstract}
Prosthetics is a field that requires a very good synergy between the dentist and the dental technician in order to create a prothetic treatment that is precise, functional and has also a good aesthetic. A good communication between the two parties is very important because, in most cases, dental technicians are located remotely and often do not see the patient. Functional analysis is an important requirement for diagnosing many different syndromes, which are usually associated with incorrect loading of the temporomandibular joint or dysfunctions. Fixed prosthesis focused on permanently attached (fixed) dentures. Such dental restorations, also called indirect restorations, include crowns, bridges (fixed prostheses), inlays, onlays and veneers. Fixed prosthesis can be used to restore one or more individual teeth, covering areas where the teeth have been lost. In general, the main advantages of fixed prosthetics compared to direct restorations are the superior strength when used in large restorations and the ability to build a tooth that has also a good aesthetic shape. As with any dental restoration, the principles used to determine Proper restoration involves taking into account the materials to be used, the extent of the tooth damage, the orientation and location of the teeth and the condition of the adjacent teeth.
\end{abstract}

\section{INTRODUCTION}

One of the most important aspects of mandibular movements is how the mandibular condyle moves along the articular surface of the skull. During the opening movement it slides from the mandibular fossa to the articular eminence in a forward (and backward) direction. The lateral pterygoid muscle appears to play a major role in this movement. When the mandible closes, the condyle moves in the opposite direction. The muscles connected directly to the condyle are not well oriented to control how the condyle performs this movement. Osborn proposed (for opening movements, but also for closing movements) that ligaments play an important role in guiding the condyle. The great freedom of movement at the joints requires that the ligaments be relatively extensible.

Furthermore, the influences of the masticatory muscles, which are the primary source of photos in the mandible during its closure, are neglected. During craniomandibular dysfunction, not only aberrant condylar pathways were observed, but also aberrant muscle needs. So, there must be a relationship between the characteristic path of the mandibular condyle during movement and the associated muscle forces.(1)

Several functional aspects are related to the position of the occlusal plane, therefore, prosthetic specialists must harmonize the dental, skeletal and soft structures of the masticatory system. Several methods have analyzed and measured the craniofacial complex and the relative positions of the occlusal and facial planes, most of these investigations using radiographic examinations that could detect the relationship between craniofacial structures in a single two-dimensional projection (usually laterolateral).(2)

Currently, 3D evaluations can be performed with computerised tomography (CBCT) and scanned reconstruction, but the method requires ionizing radiation and is not applicable in reference studies performed on healthy subjects.

It may also not be appropriate to use invasive radiological protocols on patients requiring non-surgical oral rehabilitation such as total mobile prostheses.

Therefore, the physiological position of the occlusal plane in the coronal and horizontal anatomical planes can be defined using other methods. A limited number of studies have attempted to define the 3D orientation of the occlusal plane using noninvasive procedures.

These protocols used contact tools and were based on the registration of the spatial coordinates of a limited set of marks of interest.(3)

The centred position of the mandible regarding also the gravity, at the beginning of a movement, is used as the origin of the right Cartesian coordination system, fixed to the skull (positive $\mathrm{Z}$ axis directed perpendicular to the occlusal plane of the mandible; positive $\mathrm{X}$ axis directed anteriorly; positive $\mathrm{Y}$ axis directed to the left). A similar coordinate system was attached to the mandible. At the beginning of the movement, both systems have identical positions and orientations.

The mandible may come into contact with the skull at the temporomandibular joint and occlusion points. In the present model these contacts are elastic and stable.(4)

Porion is the point on the human skull located at the upper edge of each ear canal (external auditory meatus, external acoustic meatus). It is located on the upper edge of the tragus. It is a cephalometric landmark with significance in biological anthropology and in clinical applications, such as oral and maxillofacial surgery. The porion is one of the three anatomical

${ }^{1}$ Corresponding author: Cristina Adriana Dahm Tătaru, Str. Bahluiului, Nr. 1, Sibiu, România, E-mail: drtataru@ yahoo.com, Phone: +40749 214341 Article received on 07.05.2021 and accepted for publication on 04.06.2021 


\section{CLINICAL ASPECTS}

points used to determine the Frankfurt plan. The Frankfurt Plan (also called the auriculo-orbital plan) was established at the World Congress of Anthropology in Frankfurt, Germany in 1884 and was decreed as the anatomical position of the human skull as a reference for comparative cephalometric measurements.(5)

\section{Figure no. 1. Occlusal registration using the Frankfurt plan}

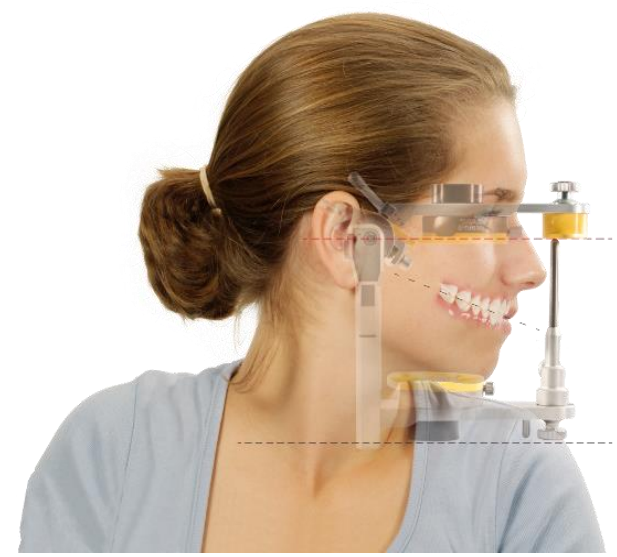

\section{AIM}

In this paper we want to present that when it comes to occlusal diagnosis in craniomandibular disorders, it is necessary to mount models in an articulator that is semiadaptable for better occlusal analysis. The data obtained after the analysis on an articulator can guide the doctor to other types of elimination in what are the dental causes of craniomandibular disorders.

\section{MATERIALS AND METHODS}

For the clinical records regarding the programming of mandibular movement parameters; at this stage, we used a RegiWax type wax produced by Girrbach Dental, this wax is intended for these types of recordings, it will be cut in the front area, more precisely in the canine area to allow the registration of position.(6)

To register the inclination angle of the slope on the articular tubercle, as well as of the condylar trajectory, it is necessary to register the propulsion movements and the "head to head" position. The patient will be asked to make two or three movements of maximum propulsion, prior to registration, and the technique will be explained, from an anterior-inferior position the mandible will be guided by the doctor in the "head to head" position.

When programming the Bennett angle on the articulator, it will be necessary to record both the lateral movement for the right and left side, as well as the "head to head" position, relative to the level of the canines on the right and left side. Prior to registration, the patient will be asked to perform two or three movements of maximum laterality on the recording side. The technique is very similar to the one presented above. This will result in two records:

- one in the frontal position with the canines on the right side to be used in the programming on the articulator for the Bennett angle on the left side

- $\quad$ another with the canines on the right side in the "end to end" position so that the Bennett angle is programmed on the right side.(7)

We made for each patient, four interarcadic registrations were made with the aim of mounting the models in the articulator.

- $\quad$ CR recorded using the sliding guide
- "head to head" for propulsion

- " "end to end" for the laterality on the right side

- "end to end" for left laterality.

Dental imprints, mandibles and jaws, together with the transfer mass materializing the position relative to the horizontal axis of jaw transfer, as well as these recordings will be used for a correct mounting in the semi-adaptable articulator of the models in the next stage, laboratory stage.

As for the programming of the angle of inclination for the slope of the articular tubercle, the RC position for the articulator will be initially unlocked in order to allow a good positioning in the recording of the maxillary model. In the lower model will be placed the wax recording for the propulsion movement.

After these stages, occlusal analysis can be reached, which offers certain advantages over the articulator, such as:

- you can change both the position of the articulator for

better visibility and the models

- premature contacts in $\mathrm{RC}$ are obvious

- interferences can be analysed in detail

- the slip from RC to IM can be appreciated more accurately.

The occlusal analysis on the articulator, as a methodology, will follow the steps of the clinical examination of the occlusion. The simulation of some movements in the articulator will have to be done with the condylar spheres in a permanent contact with the walls of the articular cavities, so that these movements are reproduced correctly.(8)

\section{RESULTS}

In this study, performed on a group of 66 patients aged between 18 and 53 years, taking into account the percentages regarding the diagnosis of ATM diseases distributed according to the sex of patients, their age and their background.

The gender distribution of the group of patients highlights the fact that women are more numerous. Of the group of 66 patients, $56 \%$ and 37 people are women, respectively, and $44 \%$ and 29 people are men, respectively.

The occlusal determination for Cranio-Mandibular Disorders has been intensely debated, the changes in the level of its ratios have been separated from the term Cranio-mandibular Disorders and have been called occlusal disharmonies.

Figure no. 2. Mandibular laterodeviation

\section{Absent}

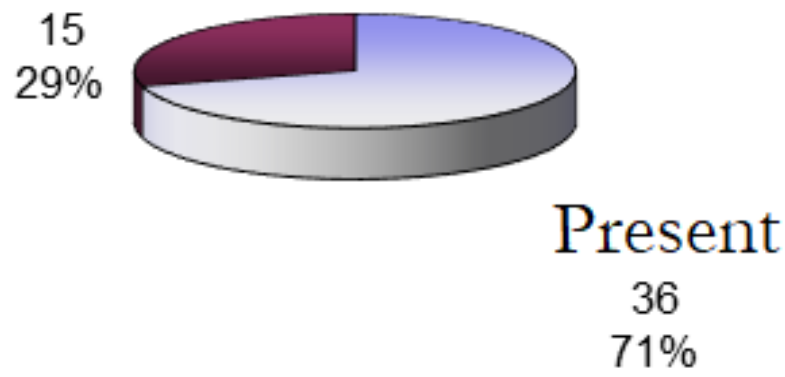

An occlusal analysis, however, if done carefully on the articulator, will be recommended in situations where the clinical examination or preliminary screening of the dental occlusion will raise certain suspicions about the existence of CranioMandibular Disorders.

Occlusal analysis in a partial adjustable articulator may confirm the data of a clinical analysis, premature interference and contact will be evident if the models are fitted correctly.(9) 


\section{CLINICAL ASPECTS}

Figure no. 3. TJM pain report

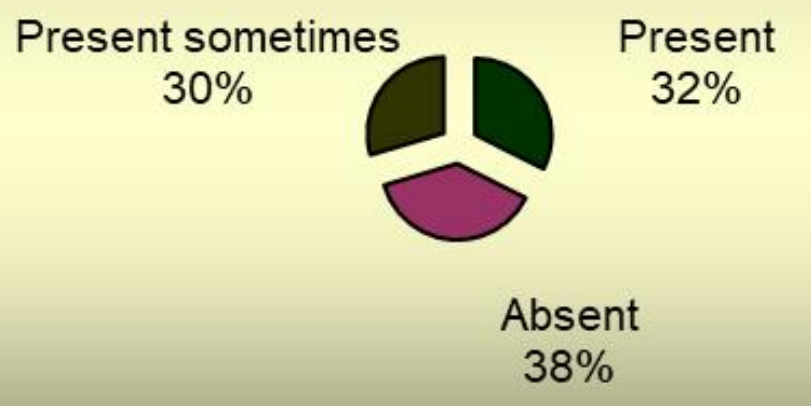

Figure no. 4. Comfort after the prosthetic treatment

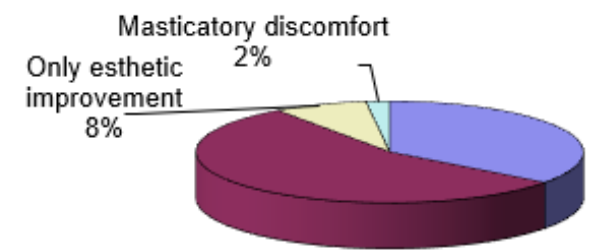

Masticatory improvement $35 \%$

Masticatory and esthetic improvement

$55 \%$

\section{DISCUSSIONS}

Occlusal analysis and mounting of models in partial or total adjustable articulators may be part of the therapeutic and diagnostic standard when it comes to prosthetic restorations, regardless of the absence or presence of the symptoms in the Cranio-Mandibular Disorders.(1)

In 1996, a consensus conference was held in New York City on the clinical attitude towards craniomandibular disorders, on the basis of which the final document denies the practical role of dental occlusion in the evolution and occurrence of craniomandibular disorders.

Disadvantages of this technique:

The temporo-mandibular joint is complex. Its articular surfaces are irregular in shape and covered with visco-elastic cartilage.

The distribution of the masticatory forces on its surface is influenced by an articular disc. In the model, this has been greatly simplified.

The articular surfaces were modeled as linear elastic structures with the usual shape.

Disc thickness was included in the elasticity estimates.

While the distribution of maxillary forces is not the subject of this study, the disc was not included as a separate structure.

For the same reason and to avoid shape-dependence coincidences, the shapes of joint surfaces have been simplified as much as possible.(10)

It was decided that a plane passing through the lower edge of the left orbit (the point called the left orbit) and the upper edge of each ear canal or external auditory canal, the Porion point, was almost parallel to the earth's surface in the position where the head is usually of a living subject. In normal subjects, both orbits and both Porion points are in one plane. However, due to the pathology, this is not always possible. The formal definition lists only the three points listed above, enough to describe a plane in three-dimensional space (5)

The mastoid index (craniometric measurement) is the distance from the Porion to the Asterion. Determining the Frankfurt plane differs between skeletal and soft tissues, the soft tissues that use the tragus as a landmark instead of the Porion.

Another name for the Frankfurt plane is the auriculoorbital plane. In order to compare human skulls with those of other species, especially hominids and primates, skulls can be studied in the Frankfurt plane. However, the Frankfurt plan is not considered to be the anatomical position for most nonprimate species.(11)

The occlusal plan has a key role in several dental and medical disciplines, such as oro-maxillofacial, aesthetic, orthognathic, plastic and reconstructive surgery, general and prosthetic dentistry. Most dental procedures involve changes in the occlusal plan. Maxillofacial surgeons, prosthetic specialists and orthodontists could change the three-dimensional (3D) orientation both in space and in relation to other craniofacial structures. $(12,13)$

\section{CONCLUSIONS}

Several functional aspects are related to the position of the occlusal plane, therefore, prosthetic specialists must harmonize the dental, skeletal and soft structures of the masticatory system.

Several methods have analysed and measured the craniofacial complex and the relative positions of the occlusal and facial planes, most of these investigations using radiographic examinations that could detect the relationship between craniofacial structures in a single two-dimensional projection (usually laterolateral).

\section{REFERENCES}

1. Garg Ak. Knochen (Biologie, Gewinnung, Transplantation in der zahnarztlichen Implantologie), Ed. Quintessence; 2008. p. 188-214.

2. Gănuță N, Bucur A, Gănuță A, Tratat de implantologie orală , Ed. National; 1996.

3. Bratu D. Bazele clinice şi tehnice ale protezării edentaţiei totale, Ed. Imprimeriei de Vest; 2004. p. 408-416.

4. Bull AMJ, Amis AA. Knee joint motion:description and measurement. In Proceedings of the IMECH E Part $\mathrm{H}$ Journal of Engineering in Medicine. 1998;212:357-372.

5. Dahm Tataru CA. Mijloace de identificare a modificarilor biomecanice ale ATM in scop proprotetic, Ed. Lucian Blaga; 2016.

6. Renouard F, Rangert BO, Risikofaktoren in der Implantologie (Klinische Diagnostik, Entscheidungsfindung und Terapie), Ed. Quintessence; 2005.

7. McMinn L, Head and neck anatomy, Wolfe Medical Publications Ltd; 1981.

8. Aldescu C. Radiologie pentru studenţi şi medici stomatologi, Ed. Polirom; 1998.

9. 9Warwick R, Williams P. Gray's anatomy 35th Edition, Longman; 1973.

10. Enciso R, Menon A, Fidaleo DA, Neumann U, and Mah J. The virtual craniofacial patient: $3 \mathrm{~d}$ jaw modeling and animation. Stud Health Technol Inform. 2003;94:65-71.

11. Nieke K, Einfurung in die Kieferortopadie, Muenchen-Baltimore; 1995. p. 224-238.

12. Schmuth G, Vardimon AD. Kieferorthopedie, Thieme; 1994. p. S 341-368.

13. Dahm Tataru CA, Morfologia dintilor permanenti umani, Ed ULBS; 2020. p. 18-22. 